\title{
Defining brain-machine interface applications by matching interface performance with device requirements
}

\author{
Oliver Tonet ${ }^{\mathrm{a}}$, Martina Marinelli ${ }^{\mathrm{a}}$, Luca Citi ${ }^{\mathrm{a}, \mathrm{b}}$, \\ Paolo Maria Rossini ${ }^{\text {c, Luca Rossini }}{ }^{\mathrm{c}, \mathrm{d}}$, Giuseppe Megali ${ }^{\mathrm{a}}$, \\ Paolo Dario ${ }^{a}$ \\ ${ }^{a}$ CRIM Lab, Scuola Superiore Sant'Anna, Pisa, Italy \\ ${ }^{\mathrm{b}}$ IMT School of Advanced Studies, Lucca, Italy \\ ${ }^{\mathrm{c}}$ Università Campus Biomedico, Rome, Italy \\ ${ }^{\mathrm{d}}$ ESA Advanced Concepts Team, Noordwijk, The Netherlands
}

\begin{abstract}
Interaction with machines is mediated by Human-Machine Interfaces (HMIs). Brainmachine interfaces (BMIs) are a particular class of HMIs and have so far been studied as a communication means for people who have little or no voluntary control of muscle activity. In this context, low-performing interfaces can be considered as prosthetic applications. On the other hand, for able-bodied users, a BMI would only be practical if conceived as an augmenting interface. In this paper a method is introduced for pointing out effective combinations of interfaces and devices for creating real-world applications. First, devices for domotics, rehabilitation and assistive robotics, and their requirements, in terms of throughput and latency, are described. Second, HMIs are classified and their performance described, still in terms of throughput and latency. Then device requirements are matched with performance of available interfaces. Simple rehabilitation and domotics devices can be easily controlled by means of BMI technology. Prosthetic hands and wheelchairs are suitable applications but do not attain optimal interactivity. Regarding humanoid robotics, the head and the trunk can be controlled by means of BMIs, while other parts require too much throughput. Robotic arms, which have been controlled by means of cortical invasive interfaces in animal studies, could be the next frontier for non-invasive BMIs. Combining smart controllers with BMIs could improve interactivity and boost BMI applications.
\end{abstract}

Key words: brain-computer interface, brain-machine interface, human-machine interface, hybrid bionic system, throughput, information transfer rate 


\section{Introduction}

\subsection{Hybrid Bionic Systems}

In everyday life, we increasingly interact with machines, such as computer, appliances, even robots. This interaction is mediated by a Human-Machine Interface (HMI). The ensemble user-interface-device, comprising both artificial and biological components, is defined as Hybrid Bionic System (HBS).

From a control system viewpoint, Fig. 1.a shows the information flow that happens as we interact with a HMI. Our intention to interact with the interface for a utilization task, e.g. grasp a knob, resides in dedicated neural networks within the brain and is translated into complex motor commands and then dispatched from the areas for motor planning and execution toward the target muscles through the cortico-spinal and peripheral nervous fibres. The results of our action are then gathered by our sensing system (eyes, touch and proprioceptive receptors, etc.), translated into sensory signals and fed back to the Central Nervous System (CNS) through the afferent pathways.

[ Figure 1 about here ]

This scenario is over-simplified, but nonetheless it allows to clarify the potentials of direct brain-machine communication. A Brain-Machine Interface (BMI), or Brain-Computer Interface (BCI), can be defined as any system able to monitor brain activity and translate a person's intentions into commands to a device. In an ideal BMI, the motor commands, instead of being sent to the physiological musculo-skeletal effectors, will reach an artificial actuator (a robot); its action on the environment will be measured by a sensing system composed of artificial sensors and the information gathered will be fed back to the CNS as natural afferent signals (see Fig. 1.b). The artificial system can be hooked in at various levels in the system above, e.g. intercepting electromyographic signals or directly stimulating the Peripheral Nervous System (PNS) for feedback, but for a true BMI, only the CNS will be interfaced; the BMI will be therefore independent from the functioning of the PNS and will be usable also by severely disabled patients. Since to date no technology can provide a viable feedback method by directly stimulating the nervous system, the usual approach is to use the natural senses, such as vision or touch, in order to dispatch relevant information to the brain.

Email address: oliver.tonet@sssup.it (Oliver Tonet). 


\subsection{Types of users}

BMIs have so far been extensively studied as a communication means for people that are affected by disabilities - such as severely advanced stages of amyotrophic lateral sclerosis (ALS) or muscular distrophies, brainstem lesions etc. - who, because of the underlying pathology, have no voluntary control of muscles (Wolpaw et al., 2002; Donoghue, 2002; Mussa-Ivaldi and Miller, 2003). In this case, despite having a normally working brain in terms of cognition and self perception, they possess no communication means with the outside world and a BMI may represent their only way to interact with other people and objects. For these cases, also BMIs of relative efficiency will represent a significant improvement in daily living abilities, so even interfaces with low bit rates can be considered as prosthetic applications. Indeed, the most skilled BCI typewriters achieve only bit rates of few letters per minute.

On the other end of the spectrum are able-bodied users. For these users, a BMI as an alternative communication device is not useful, due especially to their low bandwidth and the fact that current BMIs impose a high cognitive load, with long training periods, and do not allow the user to perform activities besides interacting with the BMI itself, to avoid the generation of artifact signals that are not directly related to the driving of the BMI. In such conditions, a BMI would only be practical if conceived as an augmenting interface, i.e. an interface that allows users to perform actions in addition to what they already can do with their normal abilities. Figure 1.c shows the human augmentation scenario, in which a user both exploits his natural neural pathways and a BMI for communicating with two or more interfaces concurrently.

\subsection{Objectives}

In this paper, within the context of HBSs, we hypothesize that performance of HMIs can be roughly compared independently from task and method and across all types of users. It is then shown how, under this hypothesis, it is possible to point out effective combinations of interfaces and devices for creating real-world applications, by using trhoughput and latency as performance measures. The paper focuses on BMI technology, but the method can be applied to all classes of HMIs. In Section 2.1 throughput and latency as performance measures for HBSs are introduced and also briefly discussed. In Section 3 typical devices for HBSs in the context of domotics, rehabilitation and assistive robotics and their requirements in terms of throughput and latency are described. In Section 4 HMIs are classified and their performance described in terms of the same parameters. In Section 5 previous requirements are finally matched with the performance of available interfaces, discussing currently fea- 
sible applications of BMI technology and possible future applications. More common human computer interfaces are used as a comparison term. The paper concludes with an overview of control factors that influence performance of HBSs and that can be exploited to boost BMI performance.

\section{Methods}

\subsection{Performance measures of $H B S$}

The performance of HBSs can be characterized by means of several numerical parameters. In this work throughput and latency are described and the used to characterize both the interface and the device components of a HBS. Throughput (also called bit rate, bandwidth, or information transfer rate) is the rate at which a communicating entity sends or receives data, i.e. the amount of data that is transferred over a period of time and is measured in bit/s. It is therefore a measure of the channel capacity of a communications link. Error probability has an influence on throughput: error correction slows down the system and wastes communication bandwidth. Latency is a time delay between the moment something is initiated, and the moment one of its effects begins (onset latency) or reaches the azimuth/nadir (peak latency). The unit of latency is time (s). Normally throughput and latency are opposed goals, because in low-latency communication more data will be wasted to control communication and to check if the other party wants to interact.

In the following, classes of interfaces and devices are characterized. For each class, a range for both throughput and latency is defined. Concerning throughput, several ways to control the devices and their communication needs have been analysed. Throughput of devices $\left(T P_{d}\right)$ was calculated as the product of the number of bits per unit command $b$ (in bit/command) and the number of commands per second $\nu$ (command/s) that have to be sent to the device to be able to control it interactively.

$$
T P_{d}=b \nu .
$$

The throughput of interfaces $\left(T P_{i}\right)$ has been calculated as the Shannon information rate in (Shannon, 1948). This definition of throughput is also popular in the literature on BCIs, having been first suggested by Wolpaw et al. (1998). In most papers $T P_{i}$ is not reported but the number of symbols, the error probability and the transfer rate (symbols/s) is stated or can be inferred. In these cases a symmetric $N$-symbol channel with symbol rate $R$ and error probability $(1-P)$ is hypothesized. Therefore the throughput $T P_{i}$ (in bit/s) has been 
calculated as:

$$
T P_{i}=R\left(\lg _{2} N+P \lg _{2} P+(1-P) \lg _{2} \frac{(1-P)}{(N-1)}\right)
$$

There are other definitions of throughput, such as the Blahut-Arimoto (used in (Santhanam et al., 2005)) and Nykopp (discussed in (Kronegg et al., 2005)). However the Shannon definition was chosen because it can be easily calculated also in studies where it is not reported and provides an acceptable measure for our needs, as will be clear in the following.

The value of latency depends on how interactive the system is intended to be, on how much feedback is needed to close the control loop and on the physiological characteristics of the fibers (nervous propagation velocity due to diameter and myelination) and the number of relays (i.e. number of synaptic interruptions) forming the loop itself. Physiological characteristics are dictating the limiting frame of the maximal time resolution of the adopted BMI. Latency is also affected by the time resolution of the technique used to retrieve information from the user, i.e. latency cannot be less than the time needed by the technique to measure the user's intent or action. It is often difficult to say which is the biggest acceptable latency for communicating with the user, so that they still feel interacting with the device and not frustrated by the long waiting time.

\subsection{Other measures}

Performance measures reported above are mainly focused on information transfer capabilities (information throughput and ability to meet deadlines). A wider range of metrics includes general measures in terms of suitability of a device for a given task and class of target users. A few of these additional parameters will be briefly introduced but neither examined in detail nor used to address the main topic of this work, i.e. the matching of interface performance with device requirements. A key parameter is the degree of invasiveness, for example because the risks related to surgical intervention inside the skull are not acceptable for augmentation devices or when a less invasive approach gives similar results. A few additional measures are related to the user-friendliness of the interface, including comfort for the user, portability, easiness of use, set-up time and need for careholders intervention. Further parameters to be considered are the degree of bidirectional control (in terms of feedback), the training requirements, the cost/effectiveness balance, and robustness to noise.

Another key point is the instantaneous and cumulative cognitive load required. The instantaneous cognitive load of the interface can make it interfere with the task at hand while the interface should work as much transparently as possi- 
ble. The cumulative cognitive load, instead, can reduce the temporal stability, i.e. for how long the user is able to drive the interface without degradation of performance, due to physical or cognitive tiring. Repetitive work in occupational settings often requires a combination of mental and physical demands, but little is known on the relationship between attention and repetitive work. Tasks which require high vigilance but low neuromuscular work, may induce a sense of effort and mental fatigue (Tomei et al., 2006); cognitive factors and mental stress may also cause muscular fatigue (Hendy et al., 1997). In attentive and cognitive tasks, it is common to observe effort and fatigue especially during goal-directed control, opposed to stimulus-directed control (Boksem et al., 2005). Lorist et al. (2000) report that aversion against a repetitive task and reaction time increase significantly after 60 minutes. This is particularly true in completely paralyzed subjects who exercise in a BMI paradigm, when the mental workload is demanding and the general situation of the subject is compromised by the background disease and the contingent effect of neuroactive drugs. Besides, efficiency is also strictly dependent on mental activity, that means that "undesired thoughts" act as interferences (Neumann et al., 2003). In this conditions, individual sessions should not be longer than few tens of minutes, with appropriate resting intervals. Moreover, external factors such as the drying of conductive gels of EEG electrodes over the period of hours, or the formation of scar tissue surrounding invasive electrodes, over time scales of weeks, also limit the total duration of BMI experiments. To compare performance over periods of such length, also temporal stability becomes an issue (Hinz et al., 2002; Neuper et al., 2005).

Concluding, some works in the literature push for a standardized framework to facilitate the direct performance comparison of BMI systems (Mason and Birch, 2003). The ideal yet subjective performance metrics could be a measure of the quality-of-life improvement for the end-user but the difficulties to exactly define it are evident.

\section{Materials: Devices}

An increasing number of electronic appliances have become more and more common in everyday environment, also thanks to portable equipment. In the field of domotics, also called home automation, HMIs are used to control devices and appliances. Domotics can contribute to a better quality of life, and can be useful for disabled and elderly people to increase their independency and autonomy. Domotics can be applied to safety and remote surveillance, to the control of doors, windows, lights, indoor climate, multimedia and communication devices, and household appliances. The concept of environmental control is interesting also for other habitats, like an office, a car or outdoor environments. 
In recent years, even robots have become more common in non-industrial environments. These robots are often called human-centered or human-friendly systems because the presence of the robot involves a close interaction between the robotic manipulation system and human beings. The most important applications of human-centered robots is rehabilitation and assistive robotics. Rehabilitation robots are in contact with the users and safety is a primary concern (Tejima, 2000). Advances in rehabilitation robotics are required by the growth of elderly population and by injured people, to assist in rehabilitation procedures and to provide new functional prostheses and orthoses. Rehabilitation robots are important for the healing of neurological diseases (Krebs et al., 2000). Neural Prosthetics, i.e. movement restoration for people with motor disabilities, is indeed another key application for BMI technology. Cortical signals have been used to control a hand orthosis (Pfurtscheller et al., 2000), with the aim to restore the connection from the brain to a paralyzed arm. A locked-in subject has also used neural signals to control a virtual hand (Kennedy et al., 2000) in the hopes that simulation would provide clues to potentially incorporating functional electrical stimulation into a BMI system to restore movement. Rehabilitation devices have hereby been grouped into several categories, namely: feeder robots, prosthetic hands (basic grasping function), wheelchairs (mobile platforms with 2 degrees of freedom (DOF)), manipulation aids (basic 6-DOF robot arm). Two or more of these devices can also be combined in complex systems. Humanoid assistive robotics deals with robots for domestic assistance, patient care, and even human augmentation. They are more complex than rehabilitation robots, have more DOFs and are supposed to be more reactive. Combinations of robot systems (hands, arms, trunk, etc.), up to a complete humanoid robot, have been considered.

Table 1 and Fig. 2 summarise the throughput-latency requirements of the above mentioned classes of devices.

[ Table 1 about here ]

[ Figure 2 about here ]

\section{Materials: Interfaces}

At a first level (see Fig. 3), interfaces are divided into cortical (the class that groups all types of BMIs) and non-cortical. Second, a distinction between invasive and non-invasive interfaces, by considering as invasive those interfaces that need skin incision, was carried out. 
[ Figure 3 about here ]

\subsection{Cortical interfaces}

Cortical interfaces (or BMIs) are all interfaces that exploit information collected from the human brain cortical relays, by various means, invasively or non-invasively.

\subsubsection{Cortical non-invasive interfaces}

Cortical non-invasive (C-NI) HMIs can measure and correctly classify specific signals of brain activity intentionally and automatically produced by the subject and translate them into device control signals. Such signals are recorded from the scalp and suffer from the limitations of their transit through the extracerebral layers (severe amplitude reduction, filtering of frequencies particularly in the high-frequency range, spreading of the generator source identification, increased contamination of the signal from the generator(s) by far-field volumetric potentials). Features commonly used in experimental studies derive from brain signals that include alterations of the electrical activity recorded through electroencephalograpy (EEG) such as mu or beta rhythms (Wolpaw et al., 1991), event-related potentials (ERPs), including the P300 and N400 evoked potential and visual evoked potentials (VEPs), either transient (to individual, low-rate stimuli) or steady-state (to prolonged trains of high-rate, repetitive stimuli) (Farwell and Donchin, 1988; Sutter, 1992; Middendorf et al., 2000; Kelly et al., 2005b), transient variations of the background rhythms, i.e. event-related (de)synchronization (ERS/ERD) (Pfurtscheller et al., 1993), slow cortical potentials (SCP) (Birbaumer et al., 1999), and activation patterns induced by mental task strategies (Curran and Stokes, 2003; Kostov and Polak, 2000). To avoid the need of skin preparation and electrolytic gels, dry recording electrodes are being studied (Mason, 2005). Today's wet electrodes are not suitable for daily use in normal living environment; dry electrodes would guarantee a good electrode/skin contact and allow acceptable signalto-noise ratio for longer session times.

Other features recorded with different modalities include neuromagnetic signals recorded through magnetoencephalography (MEG) (Tecchio et al., 1997; Georgopoulos et al., 2005), blood oxygen level-dependent (BOLD) responses recorded through functional magnetic resonance imaging (fMRI) (Weiskopf et al., 2004) and localised activity-related brain oxygenation measures recorded through near infrared spectroscopy (NIRS) (Coyle et al., 2004a).

Current cortical non-invasive HMIs are uni-directional interfaces. Brain signals 
can be used to drive a machine. Stimulating the CNS by means of non-invasive technologies, such as transcranial magnetic stimulation is not selective (Rossini et al., 1994; Rossini and Rossi, 2007). Therefore natural afferent pathways are used for communication feedback (see Fig. 1.b).

In Tab. 2 and Fig. 4 data collected and processed from the following studies are reported:

- ERP, ERD/ERS: classification of mental states, related only to motor imagery (Kauhanen et al., 2006), or including also mental tasks (such as cube rotation or calculation) (Obermaier et al., 2001; Nykopp, 2001; Lehtonen, 2002; Millán and Mouriño, 2003; Millán et al., 2004), or imagination of sensory stimulation (Dornhege et al., 2004).

- P300 evoked potentials: selection of items in a sequence, such as four-choice paradigm (Sellers et al., 2006a), or arranged into square matrices, typically of size $6 \times 6$ Donchin et al. (2000); Kaper and Ritter (2004); Kaper et al. (2004); Serby et al. (2005); Meinicke et al. (2003); Thulasidas and Guan (2005); Sellers et al. (2006b), or differently (Wang et al., 2005).

- Slow Cortical Potentials: 1-D cursor movement tasks (Birbaumer et al., 2000; Blankertz et al., 2004).

- Sensorimotor cortex rhythms: 1-D cursor movement tasks (McFarland et al., 2003; Fabiani et al., 2004; Buttfield et al., 2006) and 2-D cursor movement tasks (Wolpaw and McFarland, 2004; Fabiani et al., 2004; Geng et al., 2006; Vuckovic and Sepulveda, 2006).

- Steady-State Visual Evoked Potentials: 1-D cursor movement tasks (Middendorf et al., 2000) and nominal selection of a variable number of targets, from 2 (Kelly et al., 2005a), to 12 (Cheng et al., 2002; Wang et al., 2006).

\subsubsection{Cortical invasive interfaces}

Cortical invasive (C-I) interfaces are based on the voluntary control of the firing rate of individual neurons in the primary motor cortex. Neural signals recorded in cortical invasive interfaces range from small neuronal samples to large ensembles, including local field potentials (LFPs), spread over a single or multiple recording sites (Lebedev and Nicolelis, 2006). Commonly used intracortical electrodes are microwires (Marg and Adams, 1967), multiple electrode arrays (MEAs) (Maynard et al., 1997), and neutotrophic electrodes (Kennedy, 1989). An alternative, less invasive, recording modality is electrocorticography (ECoG) based on epidural or subdural implanted mesoelectrodes.

In humans, many experiments exploit ECoG signals measured on epilepsy patients requiring invasive monitoring of cortical activity for localization and eventual resection of an epileptogenic focus Kennedy and Bakay (1998). Also studies using MEAs are being carried out Hochberg et al. (2006). Cortical in- 
vasive interfaces have the potential to be bidirectional. However, most studies currently use them only for recording neuronal activity, relying on visual stimuli for feedback, as in the case of cortical non-invasive interfaces. Signals used in cortical invasive interfaces are usually generated by the subject through motor imagery tasks (Leuthardt et al., 2004; Graimann et al., 2004; Hochberg et al., 2006). Also, interfaces exploiting LFPs generated by non-motor imagery (e.g. in the auditory cortex) have been investigated (Wilson et al., 2006).

In Tab. 2 and Fig. 4, data collected and processed from the following studies with human subjects are reported:

- 1-D cursor movement: (Kennedy and Bakay, 1998; Kennedy et al., 2000; Leuthardt et al., 2004; Wilson et al., 2006)

- 2-D cursor movement: (Hochberg et al., 2006)

- Nominal selection of up to 4 mental states (Graimann et al., 2003, 2004)

Research on the use of cortical invasive interfaces as BMI has started on animals over three decades ago (Fetz, 1969; Humphrey et al., 1970). Indeed, being more invasive than human studies, animal experiments have shown higher performance. Data from the following animal studies, on rats, cats and monkeys, have been included, in order to demonstrate the potential of invasive technology:

- Switches: (Chapin et al., 1999; Laubach et al., 2000)

- 2-D cursor movement: (Serruya et al., 2002; Santhanam et al., 2005)

- 3-D movement of cursor and robot arm: (Wessberg et al., 2000; Taylor et al., 2002, 2003; Carmena et al., 2003)

Besides MEA, also LFP have been exploited (Bokil et al., 2006).

\subsection{Non-cortical interfaces}

Non-cortical interfaces are all interfaces that do not access the signals generated by the human cortex directly. The signals that drive the interface are measured in the peripheral nervous system, on the muscles, or are the result of muscular activity (change of body posture or physical interaction of the body with the interface).

\subsubsection{Non-cortical non-invasive interfaces}

Non-cortical non-invasive (NC-NI) interfaces, sometimes referred as Human Computer Interfaces (HCIs), are operator interfaces terminals with which users interact in order to control other devices. The interaction can include 
touch, sight, sound or any other physical or cognitive function. HCIs have been divided into classes, according to the method used to detect the control command sent to the machine.

The Switch1 class includes contact switches, i.e. keyboards, touch screens, joysticks, buttons, etc. The Switch2 class includes non-contact switches, e.g. eye blinking systems, detecting user's eye blink and using sequences of long and short blinks interpreted as semiotic messages (Grauman et al., 2001), and a camera based finger counter (Crampton and Betke, 2002). The Pointer class includes mice, laser pointers (Oh and Stuerzlinger, 2002) and interfaces built on gaze trackers (Jacob, 1990; Sibert and Jacob, 2000; Corneil and Munoz, 1996; De Silva et al., 2003; Xiao et al., 2005). The Speech class consists of dictation software and a small vocabulary automatic speech recognition system (Urban and Bajcsy, 2005; Axelrod et al., 2005). In addition to the references above, to compute the values in Tab. 2 and related figure, comparative studies of common HCIs (Fitts, 1954; Card et al., 1978; Plaisant and Sears, 1992; Hyrskykari, 1997; MacKenzie et al., 2001; Oh and Stuerzlinger, 2002; MacKenzie and Soukoreff, 2003)and the ISO Standard 9241-9 (ISO 9241-9:2000(E), 2000) were used and some data was inferred from average speeds of touchtypists (for keyboards) Smith and Cronin (1992); Khurana and Koul (2005) and telegraphers (for a single switch). A few of these interfaces, such as those based on gaze movements or eye blinking, can be used by also by severely disabled people as an alternative to BMIs.

Moreover, electromyographic (EMG) interfaces were considered. Some currently available rehabilitation devices, such as hand prostheses, exploit EMG signals recorded via surface electrodes to select different predefined commands (Zecca et al., 2004; Chan and Englehart, 2005; Englehart and Hudgins, 2003; Ajiboye and Weir, 2005). This approach offers advantages as robustness and non-invasiveness. However, it is unidirectional and the number of channels of control is limited. Values for EMG-based interfaces in Tab. 2 have been calculated from the above references.

\subsubsection{Non-cortical invasive interfaces}

The unidirectionality of EMG-based interfaces is the rationale of the recent attempts to directly connect the PNS with the artificial device by using noncortical invasive (NC-I) interfaces, i.e. invasive intra-neural interfaces. Invasiveness is obviously considered a drawback and is acceptable only if it can lead to significant and long-lasting improvements in terms of reliability, selectivity, stability of the implant.

Low invasiveness and high selectivity are not attainable at the same time. Less invasive extraneural electrodes, such as cuff and epineural electrodes, have re- 
duced selectivity. More invasive intrafascicular electrodes, such as longitudinal intrafascicular electrodes (LIFEs), MEAs and regenerative electrodes are more selective and they allow interaction with small groups of axons within a nerve fascicle. A review and comparison of different peripheral nerve interfaces can be found in (Navarro et al., 2005).

Few experiments on non-cortical invasive interfaces have been published. Apart from the above mentioned papers, some additional numerical data have been calculated from (Citi et al., 2006).

\subsection{Summary of interface performance}

Table 2, and Fig. 4 show a summary of latency and throughput values for all classes of HMIs. These values are not meant to be an exhaustive coverage of all available interfaces, nor to reproduce the performance of the interfaces in a quantitatively rigorous way. It is also worth noting that the throughputlatency boxes are a simplified graphical representation and that not all points within the boxes are reachable or have been measured in actual experiments. This holds especially for the high-throughput low-latency corner. In general, only good subjects in their best physical/mental shape can reach top performance. For most subjects, the typical performance lies more toward the center of the box.

MEG-based BCIs have recently shown performance comparable to EEG (Kauhanen et al., 2006). However MEG devices are expensive, immobile and extremely vulnerable to body-generated and urban magnetic noise, when operative outside magnetically shielded rooms. fMRI scanners are also expensive and immobile. fMRI-based BCIs, such as (Yoo et al., 2004), suffer from poor temporal discrimination due to the haemoglobin relaxation time which produce BOLD effects. On the other hand, NIRS-based BCIs, such as (Coyle et al., 2004b) are inexpensive and portable. However they suffer from very low throughput (in the order of $0.01 \mathrm{bit} / \mathrm{s}$ ). For all these reasons, BMIs based on MEG, fMRI and NIRS are not suitable to control a HBS and are not included in this study.

[ Table 2 about here ]

[ Figure 4 about here ]

Concerning BMIs, Fig. 4 clearly shows that, apart from SCP-based interfaces, in humans many BMIs have comparable values of throughput and latency, which means that there is currently no best choice for a given application. Factors that influence the choice, besides the application itself, could be the 
user, their training, and the feature extraction method. Throughputs higher than $1 \mathrm{bit} / \mathrm{s}$ are very difficult to achieve, cannot be attained by all users and sustained for a long time. Concerning scalp EEG, limitations could mainly arise from its low sensitivity, rather than from the signal classification techniques. In fact, at the current stage of technology, the generation and selection of signals detectable by EEG cannot be performed at much higher rates. This is surprisingly true also for invasive interfaces that, in human experiments, have not (yet) shown their superiority. However, this is probably due to the more pioneering status of electrodes and to the higher disability of the patients: consent to electrode implant is sought and given only in cases of very severe disabilities. However, as patients degenerate toward the locked-in state, their ability to learn and communicate with a BCI decreases (Birbaumer, 2006). In monkeys, thanks to more invasive BMIs, such systems have lower latency and higher throughputs, the latter also thanks to longer training periods. In summary, while the invasive approach can be promising for the future, open issues (Micera et al., 2006) and ethical aspects have to be investigated before they can be considered suitable for rehabilitation and for applications in able-bodied people; such concerns cannot be overcome at the present.

The main problem of cortical invasive interfaces is the limited robustness and the time-decay of their efficacy, due to the encapsulation with scar tissue around the recording area, the presence of proteins adsorbed onto electrode surface, and the micro-movements between the brain and the interface damaging the nervous system and degrading the precision of the recorded signal. Recent studies exploiting MEAs recording LFPs from a sample of hundred or thousands neurons located in the relevant cortical area, open encouraging scenarios because, even with the progressive loss of a number of neurons in contact with recording tips, the remaining amount of information is sufficient to allow the essential features of the cortical output. LFP analysis, however, has only been obtained in off-line recordings (Rickert et al., 2005; Bokil et al., 2006).

ECoG combines advantages over intracortical electrodes (no cortical invasiveness, reduced clinical risk, greater long-term stability) and EEG technology (larger amplitude of recordings, higher spatial resolution, reduced artifacts, less attenuation in the higher spectrum), while not incorporating many of their limitations (Moran, 2003). Nonetheless, ECoG is still an invasive technique requiring craniotomy and dural meningeal opening, which limits its use on specific clinical conditions.

Intraneural PNS interfaces should allow good performance in terms of throughput and latency. In fact, the results given in Tab. 2 have been calculated for the use of only one intraneural channel as in (Citi et al., 2006). The throughput should be significantly improved by the combined processing of several contacts, e.g. involving more than one of the three nerves serving the hand 
sensorimotor control (median, ulnar and radial).

\section{$5 \quad$ Results and discussions}

In this section the needs of the applications presented in Section 3 are matched with the performance of the interfaces described in Section 4, focusing on BMIs. Identifying the areas of overlap allows to define realistically which applications can be driven by means of a given BMI and also which types of BMI are suitable for a given application.

Figure 5 shows the overlap of application needs and interface performance. Matching the two parts of the figure, it is possible to point out whether the performance of a single interface meets the needs of a single application. Figure 6 is similar to Figure 5, but shows only BMIs among the interfaces and only those applications that can be potentially driven by a BMI. Figure 7 shows throughput and latencies plotted on two separate panes, providing an alternative view of the same data.

[ Figure 5 about here ]

[ Figure 6 about here ]

[ Figure 7 about here ]

At a first glance, it can be pointed out that feeder robots and domotic devices are suitable applications to be controlled by means of a BMI. This is not surprising: indeed, the control panel of domotic applications is usually a simple interface composed of switches and sliders, controls that are easily implemented by means of a BMI (Gao et al., 2003; Cincotti et al., 2006). Feeder robots have even simpler interfaces: a trigger signal is needed to activate the robot, that then executes the feeding task autonomously without further feedback from the user. Even SCP-based low-throughput BMIs can be used to control feeder robots. However, feeder robots are more easily controlled by means of puff/sip switches, which only require breath control abilities. Concerning more complex rehabilitation applications, there is an overlap for higher-performance BMIs with robotic devices that have few DOFs, i.e. BMIs can be used to control grasping with a prosthetic hand (Guger et al., 1999; Aggarwal et al., 2006) or a hand orthosis (Pfurtscheller et al., 2000; Kennedy et al., 2000; Müller-Putz et al., 2005b) and a smart wheelchair (Millán et al., 2004; Tanaka et al., 2005). All other rehabilitation applications require higher throughputs. 
Performance measured in monkeys suggest that cortical invasive interfaces could be used successfully for controlling wheelchairs and prosthetic hands with greater interactivity. With future developments, performance could increase to allow driving more complex robots, such as 3/6-DOF arms. Presently, there is no overlap in Fig. 6; this means that interactivity rates are not reached yet. However, with cortical invasive interfaces, humans have not reached the same performance as monkeys. In (Hochberg et al., 2006), the quadriplegic human subject that received the 96-MEA, was able to control a computer cursor to interact with home appliances, operate the opening and closing of a prosthetic hand and perform rudimentary actions with a multi-jointed robot arm. An interesting note is that he could perform these actions even while conversing, which suggests that invasive interfaces have greater capabilities of discriminating shared output, i.e. simultaneous orders of different content.

Concerning humanoid robotics, Fig. 6 shows that the humanoid head (2 DOF) can be interactively driven by means of BMIs. This is an interesting application that has received little attention so far. Hands-free control of 2 DOF has the potential to become a truly augmenting application, i.e. an application that could not be performed in the same way by one person alone. Practical scenarios include: a) the steering of a mobile robot-mounted camera while the user's hands are controlling the robot movements by means of other HMIs; b) the navigation through a map (scrolling the map or an image on a display) while the user's hands are controlling a keyboard and/or a mouse or are being used for gesture recognition. There is also a small overlap that shows that BMIs could be used to drive the 6-DOF humanoid trunk. This robot part was intended for slow positioning of the humanoid trunk as a starting point for fine manipulation. Indeed, the 7-DOF humanoid arm, which has a similar number of DOF, requires more trhoughput and less latency and cannot be currently driven interactively by means of a BMI.

Spellers (i.e. BCI-based typewriters) and neural cursors (i.e. BCI-operated $2-\mathrm{D}$ pointing devices) can be considered as a separate category, since they have no real minimum requirements. For patients that have no residual muscular control, a BCI speller or neural cursor can represent their only means to communicate and interact with the environment. Having no alternatives, even very slowly-responding systems are acceptable. For these reasons spellers, mostly based on P300 evoked potentials and SSVEPs, are a much investigated BCI application: (Farwell and Donchin, 1988; Wolpaw et al., 2000; Kennedy et al., 2000; Perelmouter and Birbaumer, 2000; Donchin et al., 2000; Wolpaw and McFarland, 2004; Scherer et al., 2004; Kennedy et al., 2004; Kaper and Ritter, 2004; Müller-Putz et al., 2005a; Serby et al., 2005; Thulasidas et al., 2006; Vaughan et al., 2006). EEG-based spellers reach a maximum throughput of about $1 \mathrm{bit} / \mathrm{s}$ (Kaper and Ritter, 2004), while the average is much lower (about $0.4 \mathrm{bit} / \mathrm{s}$ (Wolpaw et al., 2002)), which allows to type about 3 characters per minute. Writing can be sped up with incorporation of statistical 
language models (Ward and MacKay, 2002), or other techniques for word prediction such as T9 in cellular phones (Inverso et al., 2004), but this affects how the commands are formed, i.e. the requirement of the application, and not the interface throughput itself. Moreover, predictive methods are less robust to errors and noise.

Finally, BMIs can also be used to control devices and characters in virtual reality environments, which can be useful for device prototyping (Bayliss and Ballard, 2000; Bayliss, 2003) and video games (Mason et al., 2004; Pfurtscheller et al., 2006).

\section{Conclusions}

In this work a method to match interfaces and devices to form hybrid bionic systems has been presented. Though main is on BMI applications, the method is applicable to all kinds of HMIs and can be used in general to determine, given an application, what interface can be best suited to control it. It can also be used conversely, to find the applications that are most suited for a newly developed interface. Throughput and latency were selected as measures, since they are defined on all kinds of devices and interfaces and can easily be computed or estimated. Classification of the exponentially growing research on BMIs is a challenging task; frameworks have been proposed for objectively comparing BMI technologies (Mason et al., 2007) and the definitions of performance parameters such as bit rate are being discussed Kronegg et al. (2005). A broad formula for computing throughput has been selected: adopting another definition would have yielded slightly different values, however combinations of effective interfaces and devices would not have changed significantly.

Besides throughput and latency, there are other variables that affect performance of HBSs and that have to be taken into account for the development of a complete system. As stated in Section 2.1, BMI performance is not constant over time. On one hand, the duration of any single experimental session is limited by cognitive and physical fatigue of the user and by degradation of the BMI over time due to external factors; on the other hand mutual adaptation of user and algorithms can boost interface performance over repeated experimental sessions, by increasing the automatic component of the task and decreasing the cognitive and attentive load (Bailey et al., 2006). In fact, task repetition favour skilful performances due to progressive loss of cortical and voluntary control in favour of partly or entirely automatic behaviour. Moreover, the more automated a task is, the less is the involvement of high-level control centres, the smaller the amount of involved synapses and relays, the faster the task execution. However, BMI control efficiency reduction due to fatigue has been reported also in automatic control systems (Kennedy et al., 
2000): this is, sometimes, caused by user attempt to speed up the control (Kübler et al., 1999).

Concerning complete HBSs, it is possible to overcome limitations of the interface by improving the effectiveness of the commands sent to the device, i.e. by developing smart high-level controllers, which are able to perform parts of the tasks autonomously. HBSs with low-level controllers and no autonomous behaviour will leave all decisions to the users and will require many simple commands to be driven interactively. The commands will be simple (few bits/command) but frequent (many commands/s). On the other hand, an embedded high-level controller with a high degree of autonomy will accept complex commands from the user and then act autonomously, typically in a closed feedback loop based on data read from internal sensors. Such a controller will require complex commands from the user (many bits/command) but less often (few commands/s). Controllers with a modular degree of autonomy allow the user to switch between lower and higher levels of control, ensuring that the user is always in control of the device, but freeing them from the burden of controlling it continuously. Modulating degrees of autonomy could also be a means to overcome gaps between interface performance and application needs, by developing more deeply integrated HBS.

In conclusion, it appears as the future of research in HBSs will have many facets: not only there is room for improvement in all their individual components (user, device, interface), but also for developing more efficient strategies to make those components interact (control).

\section{Acknowledgments}

The work described here was partly supported by the Commission of the European Communities within the NEUROBOTICS Project (FP6-IST-001917, The fusion of NEUROscience and roBOTICS). Part of this research has been carried out during a joint collaboration between Scuola Superiore Sant'Anna, Università Campus Biomedico, University of Essex, and the Advanced Concepts Team of ESA (European Space Agency) in the frame of the Ariadna study no. 05/6402 (Non invasive brain-machine interfaces).

\section{References}

Aggarwal, V., Chatterjee, A., Cho, Y., Rasmussen, R., Acharya, S., Thakor, N. V., 2006. Noninvasive cortical control of a prosthetic hand with local machine control and haptic feedback. In: Ann. Meeting of Biomedical Engineering Soc (BMES 2006). Chicago, USA. 
Ajiboye, A., Weir, R., 2005. A heuristic fuzzy logic approach to EMG pattern recognition for multifunctional prosthesis control. IEEE Trans Neural Sys Rehab Eng 13 (3), 280-91.

Axelrod, S., Goel, V., Gopinath, R., Olsen, P., Visweswariah, K., 2005. Subspace constrained gaussian mixture models for speech recognition. Speech and Audio Processing, IEEE Transactions on 13 (6), 1144-1160.

Bailey, N. R., Scerbo, M. W., Freeman, F. G., Mikulka, P. J., Scott, L. A., 2006. Comparison of a brain-based adaptive system and a manual adaptable system for invoking automation. Hum Factors 48 (4), 693-709.

Bayliss, J. D., Jun 2003. Use of the evoked potential P3 component for control in a virtual apartment. IEEE Trans Neural Syst Rehabil Eng 11 (2), 113116.

Bayliss, J. D., Ballard, D. H., Jun 2000. A virtual reality testbed for braincomputer interface research. IEEE Trans Rehabil Eng 8 (2), 188-190.

Birbaumer, N., Mar 2006. Brain-computer-interface research: coming of age. Clin Neurophysiol 117 (3), 479-483.

Birbaumer, N., Ghanayim, N., Hinterberger, T., Iversen, I., Kotchoubey, B., Kbler, A., Perelmouter, J., Taub, E., Flor, H., Mar 1999. A spelling device for the paralysed. Nature 398 (6725), 297-298.

Birbaumer, N., Kbler, A., Ghanayim, N., Hinterberger, T., Perelmouter, J., Kaiser, J., Iversen, I., Kotchoubey, B., Neumann, N., Flor, H., Jun 2000. The thought translation device (TTD) for completely paralyzed patients. IEEE Trans Rehabil Eng 8 (2), 190-193.

Blankertz, B., Muller, K.-R., Curio, G., Vaughan, T., Schalk, G., Wolpaw, J., Schlogl, A., Neuper, C., Pfurtscheller, G., Hinterberger, T., Schroder, M., Birbaumer, N., 2004. The BCI competition 2003: progress and perspectives in detection and discrimination of eeg single trials. Biomedical Engineering, IEEE Transactions on 51 (6), 1044-1051.

Bokil, H. S., Pesaran, B., Andersen, R. A., Mitra, P. P., Aug 2006. A method for detection and classification of events in neural activity. IEEE Trans Biomed Eng 53 (8), 1678-1687.

Boksem, M. A. S., Meijman, T. F., Lorist, M. M., Sep 2005. Effects of mental fatigue on attention: an erp study. Brain Res Cogn Brain Res 25 (1), 107116.

Buttfield, A., Ferrez, P., Millan, J. d. R., June 2006. Towards a robust bci: error potentials and online learning. Neural Systems and Rehabilitation Engineering, IEEE Transactions on [see also IEEE Trans. on Rehabilitation Engineering] 14 (2), 164-168.

Card, S. K., English, W. K., Burr, B. J., 1978. Evaluation of mouse, ratecontrolled isometric joystick, step keys, and text keys for text selection on a crt. Ergonomics 21, 601-613.

Carmena, J. M., Lebedev, M. A., Crist, R. E., O’Doherty, J. E., Santucci, D. M., Dimitrov, D. F., Patil, P. G., Henriquez, C. S., Nicolelis, M. A. L., Nov 2003. Learning to control a brain-machine interface for reaching and grasping by primates. PLoS Biol 1 (2), E42. 
Chan, A., Englehart, K., 2005. Continuous myoelectric control for powered prostheses using hidden markov models. IEEE Trans Biomed Eng 52 (1), $121-124$.

Chapin, J. K., Moxon, K. A., Markowitz, R. S., Nicolelis, M. A., Jul 1999. Real-time control of a robot arm using simultaneously recorded neurons in the motor cortex. Nat Neurosci 2 (7), 664-670.

Cheng, M., Gao, X., Gao, S., Xu, D., 2002. Design and implementation of a brain-computer interface with high transfer rates. IEEE Trans Biomed Eng 49 (10), 1181-1186.

Cincotti, F., Aloise, F., Babiloni, F., Marciani, M., Morelli, D., Paolucci, S., Oriolo, G., Cherubini, A., Bruscino, S., Sciarra, F., Mangiola, F., Melpignano, A., Davide, F., Mattia, D., February 20-22, 2006. Brain-operated assistive devices: the aspice project. In: Biomedical Robotics and Biomechatronics, 2006. BioRob 2006. The First IEEE/RAS-EMBS International Conference on. pp. 817-822.

Citi, L., Carpaneto, J., Yoshida, K., Hoffmann, K. P., Koch, K. P., Dario, P., Micera, S., February 20-22 2006. Characterization of tfLIFE neural response for the control of a cybernetic hand. In: Proceedings of the The First IEEE/RAS-EMBS International Conference on Biomedical Robotics and Biomechatronics - BioRob. Pisa, Italy, pp. 477-482.

Corneil, B. D., Munoz, D. P., Dec 1996. The influence of auditory and visual distractors on human orienting gaze shifts. J Neurosci 16 (24), 8193-8207.

Coyle, S., Ward, T., Markham, C., McDarby, G., 2004a. On the suitability of near-infrared (NIR) systems for next-generation Brain-Computer Interfaces. Physiological Measurement 25 (4), 815-22.

Coyle, S., Ward, T., Markham, C., McDarby, G., Aug 2004b. On the suitability of near-infrared (NIR) systems for next-generation brain-computer interfaces. Physiol Meas 25 (4), 815-822.

Crampton, S., Betke, M., Oct. 2002. Finger counter: A human-computer interface. In: 7th ERCIM Workshop on User Interfaces for All. Paris, France, pp. 195-195.

Curran, E. A., Stokes, M. J., Apr 2003. Learning to control brain activity: a review of the production and control of EEG components for driving braincomputer interface (BCI) systems. Brain Cogn 51 (3), 326-336.

De Silva, G. C., Lyons, M. J., Kawato, S., Tetsutani, N., 2003. Human factors evaluation of a vision-based facial gesture interface. In: Proc. CVPR-HCI 2003.

Donchin, E., Spencer, K. M., Wijesinghe, R., Jun 2000. The mental prosthesis: assessing the speed of a P300-based brain-computer interface. IEEE Trans Rehabil Eng 8 (2), 174-179.

Donoghue, J. P., Nov 2002. Connecting cortex to machines: recent advances in brain interfaces. Nat Neurosci 5 Suppl, 1085-1088.

Dornhege, G., Blankertz, B., Curio, G., Müller, K.-R., 2004. Increase information transfer rates in BCI by CSP extension to multi-class. In: Thrun, S., Saul, L., Schölkopf, B. (Eds.), Advances in Neural Information Processing 
Systems 16. MIT Press, Cambridge, MA.

Englehart, K., Hudgins, B., 2003. A robust, real-time control scheme for multifunction myoelectric control. IEEE Trans Biomed Eng 50 (7), 848-854.

Fabiani, G., McFarland, D., Wolpaw, J., Pfurtscheller, G., Sept. 2004. Conversion of EEG activity into cursor movement by a brain-computer interface (BCI). Neural Systems and Rehabilitation Engineering, IEEE Transactions on [see also IEEE Trans. on Rehabilitation Engineering] 12 (3), 331-338.

Farwell, L. A., Donchin, E., Dec 1988. Talking off the top of your head: toward a mental prosthesis utilizing event-related brain potentials. Electroencephalogr Clin Neurophysiol 70 (6), 510-523.

Fetz, E. E., 1969. Operant conditioning of cortical unit activity. Science 163 (870), 955-958.

Fitts, P. M., 1954. The information capacity of the human motor system in controlling the amplitude of movement. Journal of Experimental Psychology 47, 381-391.

Gao, X., Xu, D., Cheng, M., Gao, S., June 2003. A bci-based environmental controller for the motion-disabled. Neural Systems and Rehabilitation Engineering, IEEE Transactions on [see also IEEE Trans. on Rehabilitation Engineering] 11 (2), 137-140.

Geng, T., Gan, J. Q., Dyson, M., Tsui, C. S., Sepulveda, F., 2006. EEG-based synchronous parallel bci. In: Proc. MAIA Workshop on Brain Computer Interfaces.

Georgopoulos, A., Langheim, F., Leuthold, A., Merkle, A., Jul 2005. Magnetoencephalographic signals predict movement trajectory in space. Exp Brain Res, 1-4.

Graimann, B., Huggins, J. E., Levine, S. P., Pfurtscheller, G., Jun 2004. Toward a direct brain interface based on human subdural recordings and wavelet-packet analysis. IEEE Trans Biomed Eng 51 (6), 954-962.

Graimann, B., Huggins, J. E., Schlgl, A., Levine, S. P., Pfurtscheller, G., Sep 2003. Detection of movement-related desynchronization patterns in ongoing single-channel electrocorticogram. IEEE Trans Neural Syst Rehabil Eng 11 (3), 276-281.

Grauman, K., Betke, M., Gips, J., Bradski, G. R., 2001. Communication via eye blinks - detection and duration analysis in real time. In: Proceedings of the IEEE Computer Society Conference on Computer Vision and Pattern Recognition. Vol. 1. pp. I-1010-I-1017.

Guger, C., Harkam, W., Hertnaes, C., Pfurtscheller, G., 1999. Prosthetic control by an EEG-based brain-computer interface (BCI). In: Proceedings of AAATE 5th European Conference for the Advancement of Assistive Technology. Düsseldorf, Germany.

Hendy, K. C., Liao, J., Milgram, P., Mar 1997. Combining time and intensity effects in assessing operator information-processing load. Hum Factors 39 (1), 30-47.

Hinz, A., Hueber, B., Schreinicke, G., Seibt, R., Apr 2002. Temporal stability of psychophysiological response patterns: concepts and statistical tools. Int 
J Psychophysiol 44 (1), 57-65.

Hochberg, L. R., Serruya, M. D., Friehs, G. M., Mukand, J. A., Saleh, M., Caplan, A. H., Branner, A., Chen, D., Penn, R. D., Donoghue, J. P., Jul 2006. Neuronal ensemble control of prosthetic devices by a human with tetraplegia. Nature 442 (7099), 164-171.

Humphrey, D. R., Schmidt, E. M., Thompson, W. D., 1970. Predicting measures of motor performance from multiple cortical spike trains. Science 170 (959), 758-762.

Hyrskykari, A., 1997. Gaze control as an input device. In: Proc. of ACHCI'97. Inverso, S. A., Hawes, N., Kelleher, J., Allen, R., Haase, K., September 2004. Think and spell: Context-sensitive predictive text for an ambiguous keyboard brain-computer interface speller. Biomedizinische Technik 49 (1), 5354 .

ISO 9241-9:2000(E), 2000. Ergonomic requirements for office work with vdts - part 9 - requirements for non-keyboard input devices. Tech. rep., International Standards Organization.

Jacob, R. J. K., 1990. What you look at is what you get: Eye movement-based interaction techniques. In: Proc. of CHI'90: ACM Conference on Human Factors in Computing Systems. Addison-Wesley/ACM Press, pp. 11-18.

Kaper, M., Meinicke, P., Grossekathoefer, U., Lingner, T., Ritter, H., Jun 2004. BCI Competition 2003-Data set IIb: support vector machines for the P300 speller paradigm. IEEE Trans Biomed Eng 51 (6), 1073-1076.

Kaper, M., Ritter, H., 2004. Generalizing to new subjects in brain-computer interfacing. In: Proc. Engineering in Medicine and Biology Society Conference - EMBC. Vol. 6. pp. 4363-4366.

Kauhanen, L., Nykopp, T., Lehtonen, J., Jylnki, P., Heikkonen, J., Rantanen, P., Alaranta, H., Sams, M., Jun 2006. EEG and MEG brain-computer interface for tetraplegic patients. IEEE Trans Neural Syst Rehabil Eng 14 (2), 190-193.

Kelly, S., Lalor, E., Finucane, C., McDarby, G., Reilly, R., 2005a. Visual spatial attention control in an independent brain-computer interface. IEEE Trans Biomed Eng 52 (9), 1588-1596.

Kelly, S., Lalor, E., Reilly, R., Foxe, J., 2005b. Visual spatial attention tracking using high-density SSVEP data for independent brain-computer communication. IEEE Transactions on Neural System and Rehabilitation Engineering 13 (2), 172-178.

Kennedy, P. R., Sep 1989. The cone electrode: a long-term electrode that records from neurites grown onto its recording surface. J Neurosci Methods 29 (3), 181-193.

Kennedy, P. R., Bakay, R. A., Jun 1998. Restoration of neural output from a paralyzed patient by a direct brain connection. Neuroreport 9 (8), 17071711.

Kennedy, P. R., Bakay, R. A., Moore, M. M., Adams, K., Goldwaithe, J., Jun 2000. Direct control of a computer from the human central nervous system. IEEE Trans Rehabil Eng 8 (2), 198-202. 
Kennedy, P. R., Kirby, M. T., Moore, M. M., King, B., Mallory, A., Sep 2004. Computer control using human intracortical local field potentials. IEEE Trans Neural Syst Rehabil Eng 12 (3), 339-344.

Khurana, U., Koul, A., may 2005. Text compression and superfast searching. ArXiv Computer Science e-prints arXiv:cs/0505056, 11.

Kostov, A., Polak, M., Jun 2000. Parallel man-machine training in development of EEG-based cursor control. IEEE Trans Rehabil Eng 8 (2), 203-205.

Krebs, H. I., Volpe, B. T., Aisen, M. L., Hogan, N., 2000. Increasing productivity and quality of care: Robot-aided neuro-rehabilitation. J Rehabil Res Dev 37 (6), 639-652.

Kronegg, J., Voloshynovskiy, S., Pun, T., 2005. Analysis of bit-rate definitions for Brain-Computer Interfaces. In: In Proceedings of the 2005 Int. Conf. on Human-Computer Interaction.

Kübler, A., Kotchoubey, B., Hinterberger, T., Ghanayim, N., Perelmouter, J., Schauer, M., Fritsch, C., Taub, E., Birbaumer, N., Jan 1999. The thought translation device: a neurophysiological approach to communication in total motor paralysis. Exp Brain Res 124 (2), 223-232.

Laubach, M., Wessberg, J., Nicolelis, M. A., Jun 2000. Cortical ensemble activity increasingly predicts behaviour outcomes during learning of a motor task. Nature 405 (6786), 567-571.

Lebedev, M. A., Nicolelis, M. A., 2006. Brain-machine interfaces: past, present and future. Trends Neurosci 29 (9), 536-46.

Lehtonen, J., 2002. EEG-based brain computer interfaces. Master's thesis, Helsinki Univesrsity of Technology, Dept. of Electrical and Comm. Eng.

Leuthardt, E. C., Schalk, G., Wolpaw, J. R., Ojemann, J. G., Moran, D. W., Jun 2004. A brain-computer interface using electrocorticographic signals in humans. J Neural Eng 1 (2), 63-71.

Lorist, M. M., Klein, M., Nieuwenhuis, S., Jong, R. D., Mulder, G., Meijman, T. F., Sep 2000. Mental fatigue and task control: planning and preparation. Psychophysiology 37 (5), 614-625.

MacKenzie, I. S., Soukoreff, R. W., 2003. Card, english, and burr (1978): 25 years later. In: Proc. CHI 2003 Conference on Human Factors in Computing Systems. pp. 760-761.

MacKenzie, S. I., Kauppinen, T., Silfverberg, M., 2001. Accuracy measures for evaluating computer pointing devices. In: Proc. CHI 2001 Conference on Human Factors in Computing Systems. pp. 9-16.

Marg, E., Adams, J. E., Sep 1967. Indwelling multiple micro-electrodes in the brain. Electroencephalogr Clin Neurophysiol 23 (3), 277-280.

Mason, S. G., 2005. Dry electrode technology: What exists and what is under development? Tech. rep., Neil Squire Society.

Mason, S. G., Bashashati, A., Fatourechi, M., Navarro, K. F., Birch, G. E., Feb 2007. A comprehensive survey of brain interface technology designs. Ann Biomed Eng 35 (2), 137-169.

URL http://dx.doi.org/10.1007/s10439-006-9170-0

Mason, S. G., Birch, G. E., Mar 2003. A general framework for brain-computer 
interface design. IEEE Trans Neural Syst Rehabil Eng 11 (1), 70-85.

Mason, S. G., Bohringer, R., Borisoff, J. F., Birch, G. E., 2004. Real-time control of a video game with a direct brain-computer interface. J Clin Neurophysiol 21 (6), 404-408.

Maynard, E. M., Nordhausen, C. T., Normann, R. A., Mar 1997. The Utah intracortical Electrode Array: a recording structure for potential braincomputer interfaces. Electroencephalogr Clin Neurophysiol 102 (3), 228239.

McFarland, D. J., Sarnacki, W. A., Wolpaw, J. R., Jul 2003. Brain-computer interface (BCI) operation: optimizing information transfer rates. Biol Psychol 63 (3), 237-251.

Meinicke, P., Kaper, M., Hoppe, F., Heumann, M., Ritter, H., 2003. Improving transfer rates in brain computer interfacing: A case study. In: Thrun, S., Becker, S., Obermayer, K. (Eds.), Proc. Advances in Neural Information Processing Systems 15. pp. 1107-1114.

Micera, S., Carrozza, M., Beccai, L., Vecchi, F., Dario, P., 2006. Hybrid bionic systems for the replacement of hand function. Proc IEEE 94 (9), 1752-1762.

Middendorf, M., McMillan, G., Calhoun, G., Jones, K. S., 2000. Braincomputer interfaces based on the steady-state visual-evoked response. IEEE Trans Rehabil Eng 8 (2), 211-214.

Millán, J. d. R., Mouriño, J., 2003. Asynchronous BCI and local neural classifiers: An overview of the Adaptive Brain Interface project. Neural Systems and Rehabilitation Engineering, IEEE Transactions on 11 (2), 159-161.

Millán, J. d. R., Renkens, F., Mouriño, J., Gerstner, W., Jun 2004. Noninvasive brain-actuated control of a mobile robot by human EEG. IEEE Trans Biomed Eng 51 (6), 1026-1033.

Moran, D. W., 2003. Electrocorticographic (ECoG) control of brain-computer interfaces. In: Proc. WTEC Brain-Computer Interface Workshop. pp. 21-25.

Müller-Putz, G. R., Scherer, R., Brauneis, C., Pfurtscheller, G., Dec 2005a. Steady-state visual evoked potential (SSVEP)-based communication: impact of harmonic frequency components. J Neural Eng 2 (4), 123-130.

Müller-Putz, G. R., Scherer, R., Pfurtscheller, G., Rupp, R., 2005b. EEGbased neuroprosthesis control: a step towards clinical practice. Neurosci Lett 382 (1-2), 169-174.

Mussa-Ivaldi, F. A., Miller, L. E., Jun 2003. Brain-machine interfaces: computational demands and clinical needs meet basic neuroscience. Trends Neurosci 26 (6), 329-334.

Navarro, X., Krueger, T. B., Lago, N., Micera, S., Stieglitz, T., Dario, P., Sep 2005. A critical review of interfaces with the peripheral nervous system for the control of neuroprostheses and hybrid bionic systems. J Peripher Nerv Syst 10 (3), 229-258.

Neumann, N., Kbler, A., Kaiser, J., Hinterberger, T., Birbaumer, N., 2003. Conscious perception of brain states: mental strategies for brain-computer communication. Neuropsychologia 41 (8), 1028-1036.

Neuper, C., Grabner, R. H., Fink, A., Neubauer, A. C., Jul 2005. Long-term 
stability and consistency of EEG event-related (de-)synchronization across different cognitive tasks. Clin Neurophysiol 116 (7), 1681-1694.

Nykopp, T., 2001. Statistical modelling issues for the adaptive brain interface. Master's thesis, Helsinki Univesrsity of Technology, Dept. of Electrical and Comm. Eng.

Obermaier, B., Neuper, C., Guger, C., Pfurtscheller, G., Sep 2001. Information transfer rate in a five-classes brain-computer interface. IEEE Trans Neural Syst Rehabil Eng 9 (3), 283-288.

Oh, J.-Y., Stuerzlinger, W., May 2002. Laser Pointers as Collaborative Pointing Devices. In: Proc. Graphics Interface. pp. 141-150.

Perelmouter, J., Birbaumer, N., Jun 2000. A binary spelling interface with random errors. IEEE Trans Rehabil Eng 8 (2), 227-232.

Pfurtscheller, G., Flotzinger, D., Kalcher, J., 1993. Brain-computer interface: a new communication device for handicapped persons. J Microcomputer App 16 (3), 293-299.

Pfurtscheller, G., Guger, C., Mller, G., Krausz, G., Neuper, C., Oct 2000. Brain oscillations control hand orthosis in a tetraplegic. Neurosci Lett 292 (3), 211-214.

Pfurtscheller, G., Leeb, R., Keinrath, C., Friedman, D., Neuper, C., Guger, C., Slater, M., Feb 2006. Walking from thought. Brain Res 1071 (1), 145-152.

Plaisant, C., Sears, A., 1992. Touchscreen interfaces for alphanumeric data entry. In: Proc. of the Human Factors Society - 36th Annual Meeting. Atlanta, GA, USA.

Rickert, J., de Oliveira, S. C., Vaadia, E., Aertsen, A., Rotter, S., Mehring, C., Sep 2005. Encoding of movement direction in different frequency ranges of motor cortical local field potentials. J Neurosci 25 (39), 8815-8824.

Rossini, P. M., Barker, A. T., Berardelli, A., Caramia, M. D., Caruso, G., Cracco, R. Q., Dimitrijević, M. R., Hallett, M., Katayama, Y., Lcking, C. H., 1994. Non-invasive electrical and magnetic stimulation of the brain, spinal cord and roots: basic principles and procedures for routine clinical application. report of an IFCN committee. Electroencephalogr Clin Neurophysiol 91 (2), 79-92.

Rossini, P. M., Rossi, S., Feb 2007. Transcranial magnetic stimulation: diagnostic, therapeutic, and research potential. Neurology 68 (7), 484-488.

Santhanam, G., Ryu, S., Yu, B., Afshar, A., Shenoy, K., March 16-19, 2005. A high performance neurally-controlled cursor positioning system. In: Neural Engineering, 2005. Conference Proceedings. 2nd International IEEE EMBS Conference on. pp. 494-500.

Scherer, R., Müller, G. R., Neuper, C., Graimann, B., Pfurtscheller, G., Jun 2004. An asynchronously controlled EEG-based virtual keyboard: improvement of the spelling rate. IEEE Trans Biomed Eng 51 (6), 979-984.

Sellers, E. W., Kbler, A., Donchin, E., 2006a. Brain-computer interface research at the university of south florida cognitive psychophysiology laboratory: the P300 speller. IEEE Trans Neural Syst Rehabil Eng 14 (2), 221-224.

Sellers, E. W., Krusienski, D. J., McFarland, D. J., Vaughan, T. M., Wolpaw, 
J. R., Oct 2006b. A P300 event-related potential brain-computer interface (BCI): the effects of matrix size and inter stimulus interval on performance. Biol Psychol 73 (3), 242-252.

Serby, H., Yom-Tov, E., Inbar, G. F., Mar 2005. An improved P300-based brain-computer interface. IEEE Trans Neural Syst Rehabil Eng 13 (1), 8998.

Serruya, M. D., Hatsopoulos, N. G., Paninski, L., Fellows, M. R., Donoghue, J. P., Mar 2002. Instant neural control of a movement signal. Nature 416 (6877), 141-142.

Shannon, C., 1948. A mathematical theory of communication. Bell System Technical Journal 27, 379-423 and 623-656.

Sibert, L. E., Jacob, R. J. K., 2000. Evaluation of eye gaze interaction. In: CHI. pp. 281-288.

Smith, W., Cronin, D., 1992. Ergonomic test of the kinesis contoured keyboard. Tech. rep., Global Ergonomic Technologies, Inc.

Sutter, E. E., 1992. The brain response interface: communication through visually-induced electrical brain responses. J Microcomputer App 15 (1), 31-45.

Tanaka, K., Matsunaga, K., Wang, H., Aug. 2005. Electroencephalogrambased control of an electric wheelchair. Robotics, IEEE Transactions on [see also Robotics and Automation, IEEE Transactions on] 21 (4), 762-766.

Taylor, D., Tillery, S., Schwartz, A., June 2003. Information conveyed through brain-control: cursor versus robot. Neural Systems and Rehabilitation Engineering, IEEE Transactions on [see also IEEE Trans. on Rehabilitation Engineering] 11 (2), 195-199.

Taylor, D. M., Tillery, S. I. H., Schwartz, A. B., Jun 2002. Direct cortical control of 3D neuroprosthetic devices. Science 296 (5574), 1829-1832.

Tecchio, F., Rossini, P. M., Pizzella, V., Cassetta, E., Romani, G. L., Aug 1997. Spatial properties and interhemispheric differences of the sensory hand cortical representation: a neuromagnetic study. Brain Res 767 (1), 100-108.

Tejima, N., 2000. Rehabilitation robotics : a review. Advanced robotics 14 (7), $551-564$.

Thulasidas, M., Guan, C., 2005. Optimization of bci speller based on P300 potential. Conf Proc IEEE Eng Med Biol Soc 5, 5396-5399.

Thulasidas, M., Guan, C., Wu, J., Mar 2006. Robust classification of EEG signal for brain-computer interface. IEEE Trans Neural Syst Rehabil Eng 14 (1), 24-29.

Tomei, G., Cinti, M. E., Cerratti, D., Fioravanti, M., 2006. [attention, repetitive works, fatigue and stress]. Ann Ig 18 (5), 417-429.

Urban, M., Bajcsy, P., 2005. Fusion of voice, gesture, and human-computer interface controls for remotely operated robot. In: The Eighth International Conference on Information Fusion.

Vaughan, T., McFarland, D., Schalk, G., Sarnacki, W., Krusienski, D., Sellers, E., Wolpaw, J., June 2006. The Wadsworth BCI research and development program: at home with BCI. Neural Systems and Rehabilitation 
Engineering, IEEE Transactions on [see also IEEE Trans. on Rehabilitation Engineering] 14 (2), 229-233.

Vuckovic, A., Sepulveda, F., 2006. EEG gamma band information in cuebased single trial classification of four movements about right wrist. In: Proc. MAIA Workshop on Brain Computer Interfaces.

Wang, C., Guan, C., Zhang, H., 2005. P300 brain-computer interface design for communication and control applications. Conf Proc IEEE Eng Med Biol Soc 5, 5400-5403.

Wang, Y., Wang, R., Gao, X., Hong, B., Gao, S., Jun 2006. A practical vepbased brain-computer interface. IEEE Trans Neural Syst Rehabil Eng 14 (2), 234-239.

Ward, D. J., MacKay, D. J. C., Aug 2002. Artificial intelligence: fast hands-free writing by gaze direction. Nature 418 (6900), 838.

Weiskopf, N., Mathiak, K., Bock, S. W., Scharnowski, F., Veit, R., Grodd, W., Goebel, R., Birbaumer, N., Jun 2004. Principles of a brain-computer interface (BCI) based on real-time functional magnetic resonance imaging (fMRI). IEEE Transactions on Biomedical Engineering 51 (6), 966-970.

Wessberg, J., Stambaugh, C. R., Kralik, J. D., Beck, P. D., Laubach, M., Chapin, J. K., Kim, J., Biggs, S. J., Srinivasan, M. A., Nicolelis, M. A., Nov 2000. Real-time prediction of hand trajectory by ensembles of cortical neurons in primates. Nature 408 (6810), 361-365.

Wilson, J. A., Felton, E. A., Garell, P. C., Schalk, G., Williams, J. C., Jun 2006. Ecog factors underlying multimodal control of a brain-computer interface. IEEE Trans Neural Syst Rehabil Eng 14 (2), 246-250.

Wolpaw, J. R., Birbaumer, N., McFarland, D. J., Pfurtscheller, G., Vaughan, T. M., Jun 2002. Brain-computer interfaces for communication and control. Clin Neurophysiol 113 (6), 767-791.

Wolpaw, J. R., McFarland, D. J., Dec 2004. Control of a two-dimensional movement signal by a noninvasive brain-computer interface in humans. Proc Natl Acad Sci U S A 101 (51), 17849-17854.

Wolpaw, J. R., McFarland, D. J., Neat, G. W., Forneris, C. A., Mar 1991. An EEG-based brain-computer interface for cursor control. Electroencephalogr Clin Neurophysiol 78 (3), 252-259.

Wolpaw, J. R., McFarland, D. J., Vaughan, T. M., Jun 2000. Brain-Computer Interface research at the Wadsworth Center. IEEE transactions on rehabilitation engineering 8 (2), 222-226.

Wolpaw, J. R., Ramoser, H., McFarland, D. J., Pfurtscheller, G., 1998. EEGbased communication: improved accuracy by response verification. IEEE Trans Rehabil Eng 6, 326-333.

Xiao, M., Hyppolite, J., Pomplun, M., Sunkara, S., Carbone, E., 2005. Compensating for the eye-hand span improves gaze control in human-computer interfaces. In: Proc. HCII 2005 - 11th International Conference on HumanComputer Interaction.

Yoo, S.-S., Fairneny, T., Chen, N.-K., Choo, S.-E., Panych, L. P., Park, H., Lee, S.-Y., Jolesz, F. A., Jul 2004. Brain-computer interface using fmri: 
spatial navigation by thoughts. Neuroreport 15 (10), 1591-1595.

Zecca, M., Cappiello, G., Sebastiani, F., Roccella, S., Vecchi, F., Carrozza, M., Dario, P., 2004. Experimental analysis of the proprioceptive and exteroceptive sensors of an underactuated prosthetic hand. Lect Notes Contr Inf 306, $233-242$. 


\section{Figure Captions}

Figure 1: Schematization of human-machine interaction loops: a) the natural interaction; b) brain-machine interaction; c) human augmentation.

Figure 2: Graphical representation of the data in Tab. 1: throughput and latency requirements of domotics, rehabilitation and robotic devices. Thick lines represent classes of devices, thin lines represent subclasses.

Figure 3: Classification of human-machine interfaces. Examples of signal acquisition techniques and of acquired signals are listed for each class.

Figure 4: Graphical representation of the data in Tab. 2: Maximum and minimum values of throughput and latency for all classes of HMIs. Thick lines represent classes of devices, thin lines represent subclasses.

Figure 5: Match of devices and all classes of interfaces. The upper part of the figure focuses on devices, plotting rectangles for each device class, while the performance of all interfaces is drawn as a gray area on the background; the lower part of the figure focuses on interfaces and shows the opposite. Matching the two parts allows to find out if a device can be driven by a given interface.

Figure 6: Match of devices and all types of brain-machine interfaces. The upper part of the figure focuses on devices, plotting rectangles for each BMI class, while the performance of interfaces is drawn as a gray area on the background; the lower part of the figure focuses on interfaces and shows the opposite. Only the devices suitable to be driven by a BMI have been plotted.

Figure 7: Box plots showing throughput (left) and latency (right) of devices and interfaces, providing an alternative representation of data in Fig. 5. Moreover, median and quartiles are plotted for all classes. The single experimental values from the papers listed in the text are shown on the box plots as dots. 


\section{Table Captions}

Table 1: Throughput and latency requirements of domotics, rehabilitation and robotic devices. The provided minimum and maximum values ensure interactive behaviour for a variety of users and applications.

Table 2: Maximum and minimum values of throughput and latency for all classes of HMIs. Values have been computed from the studies listed in the text. 

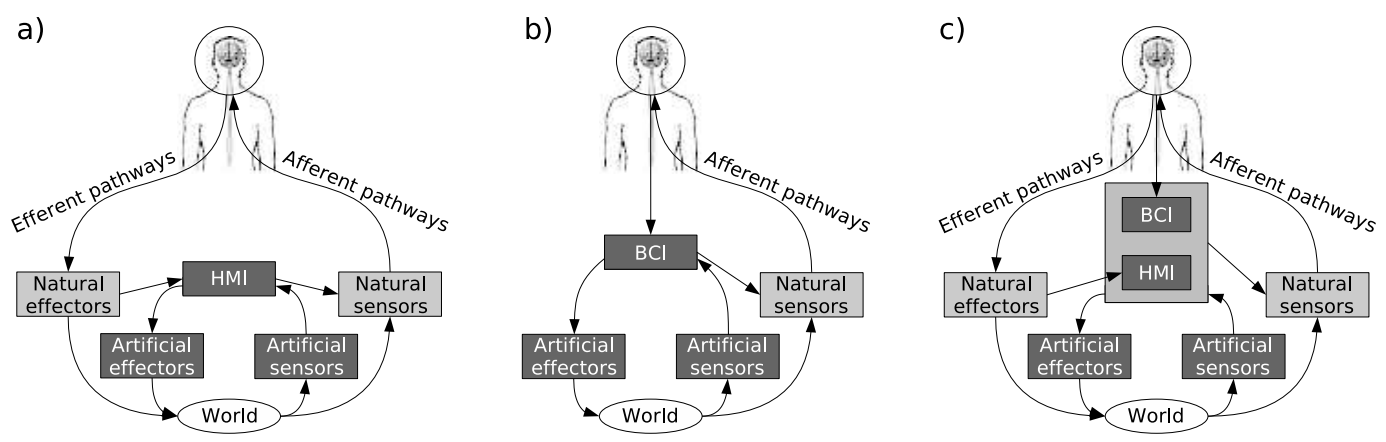

Fig. 1. 


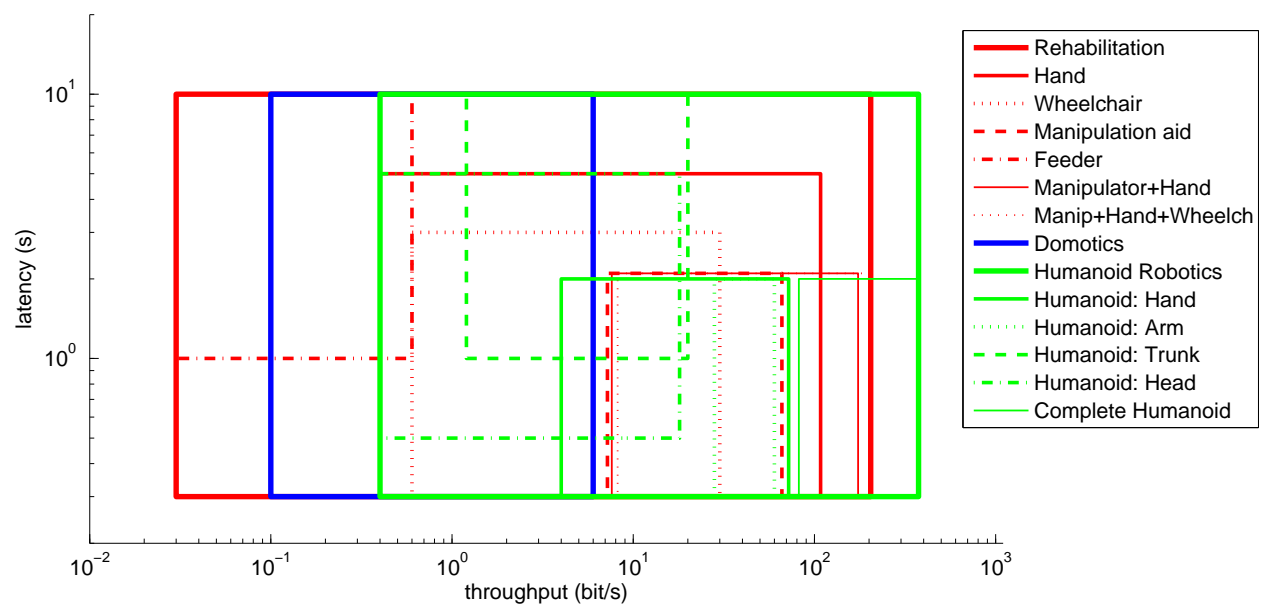

Fig. 2. 


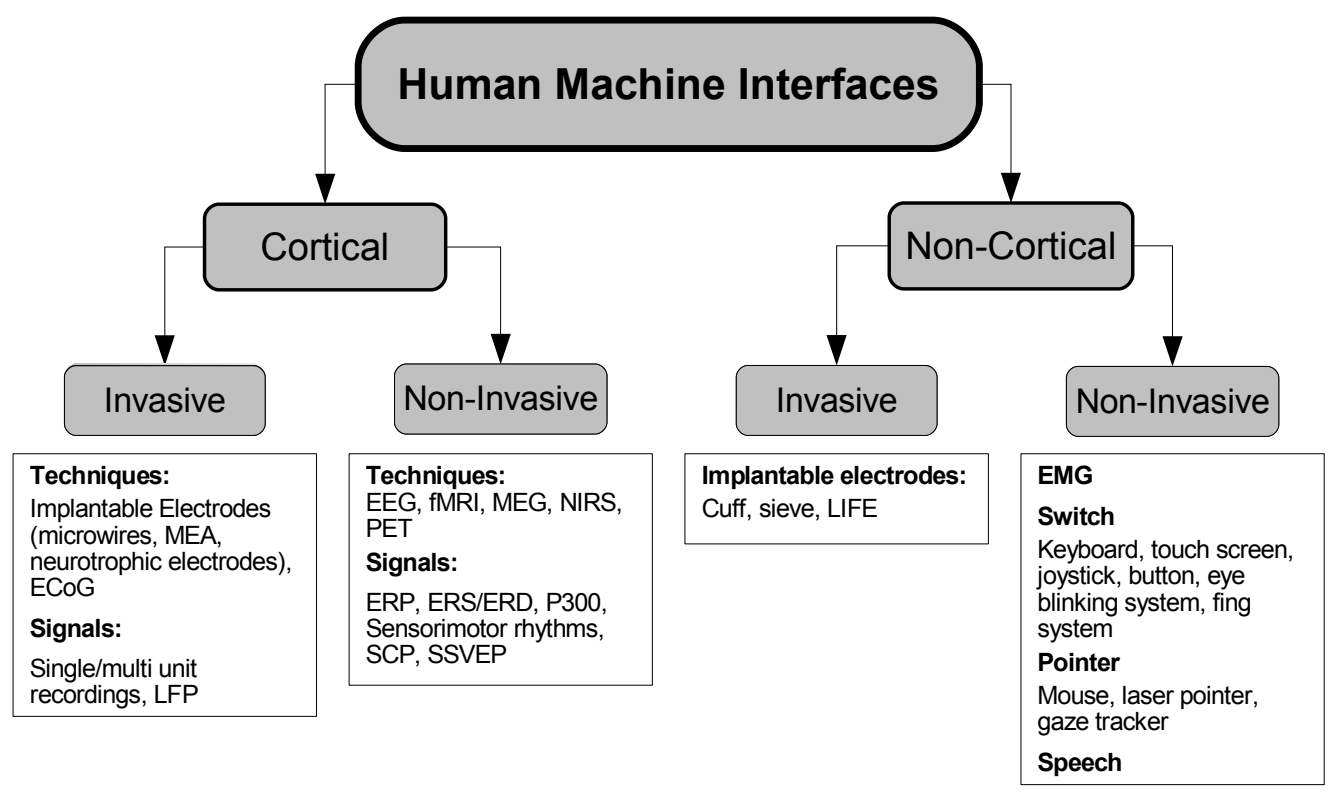

Fig. 3. 


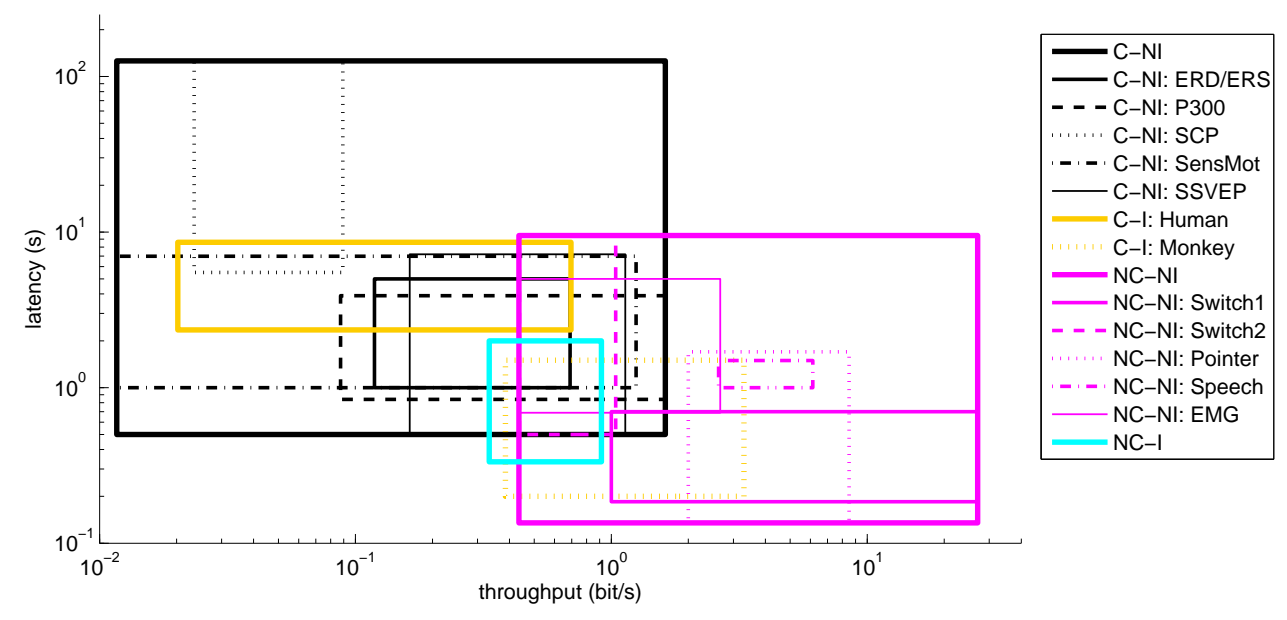

Fig. 4. 

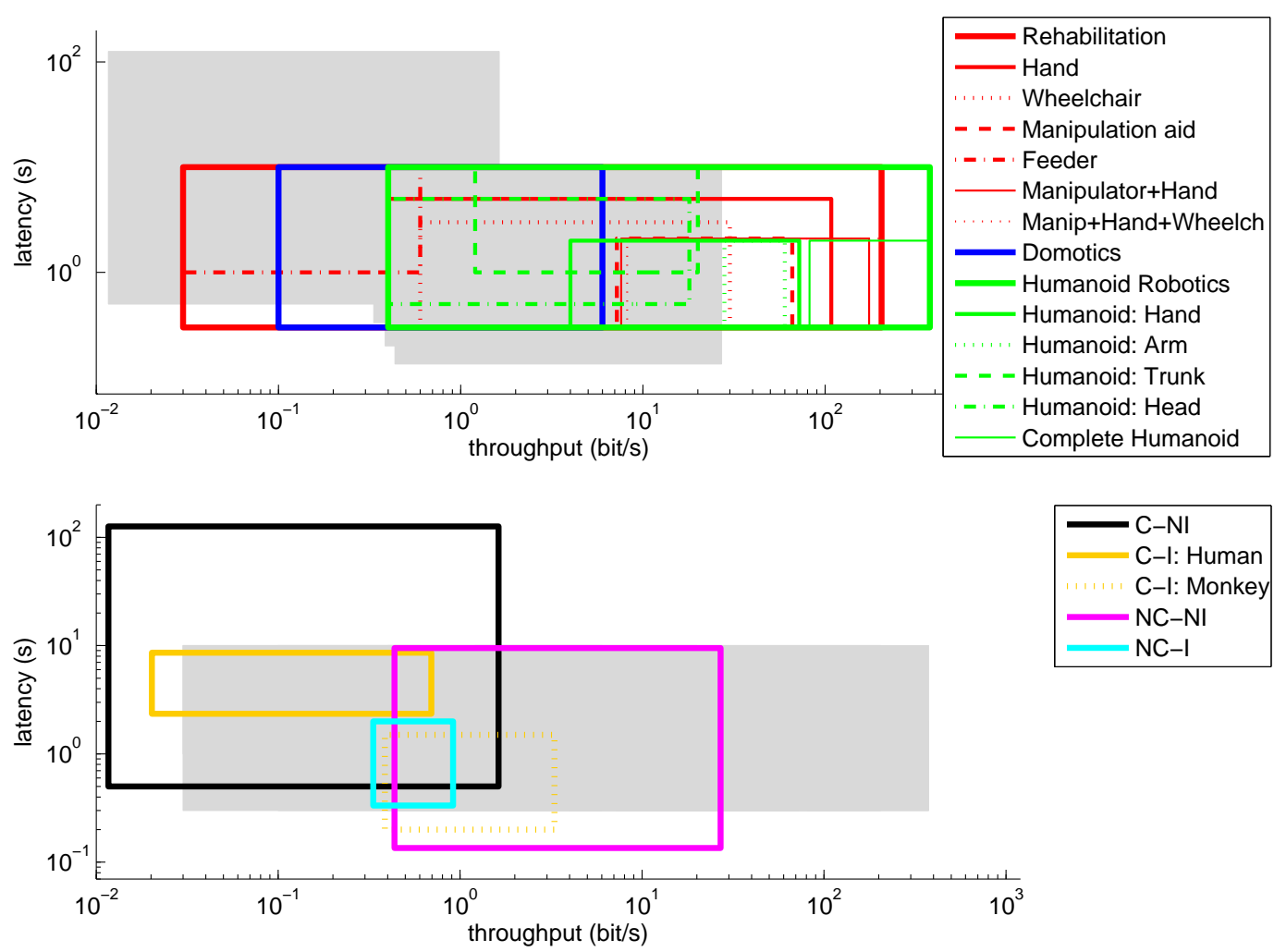

Fig. 5 . 

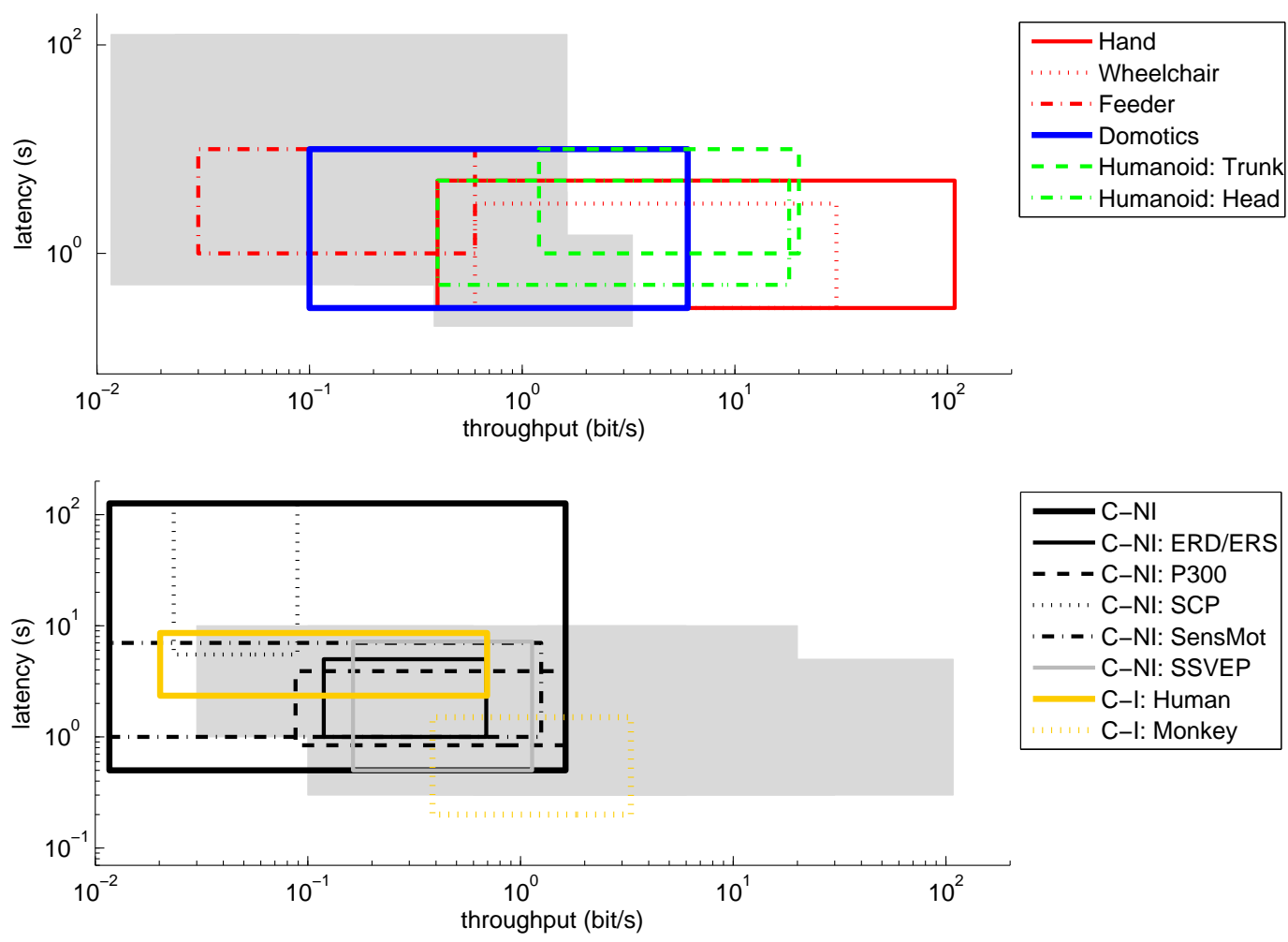

C-NI: ERD/ERS

- - - C-NI: P300

C-NI: SCP

- - C-NI: SensMot

C-NI: SSVEP

C-I: Human

C-I: Monkey

Fig. 6 . 

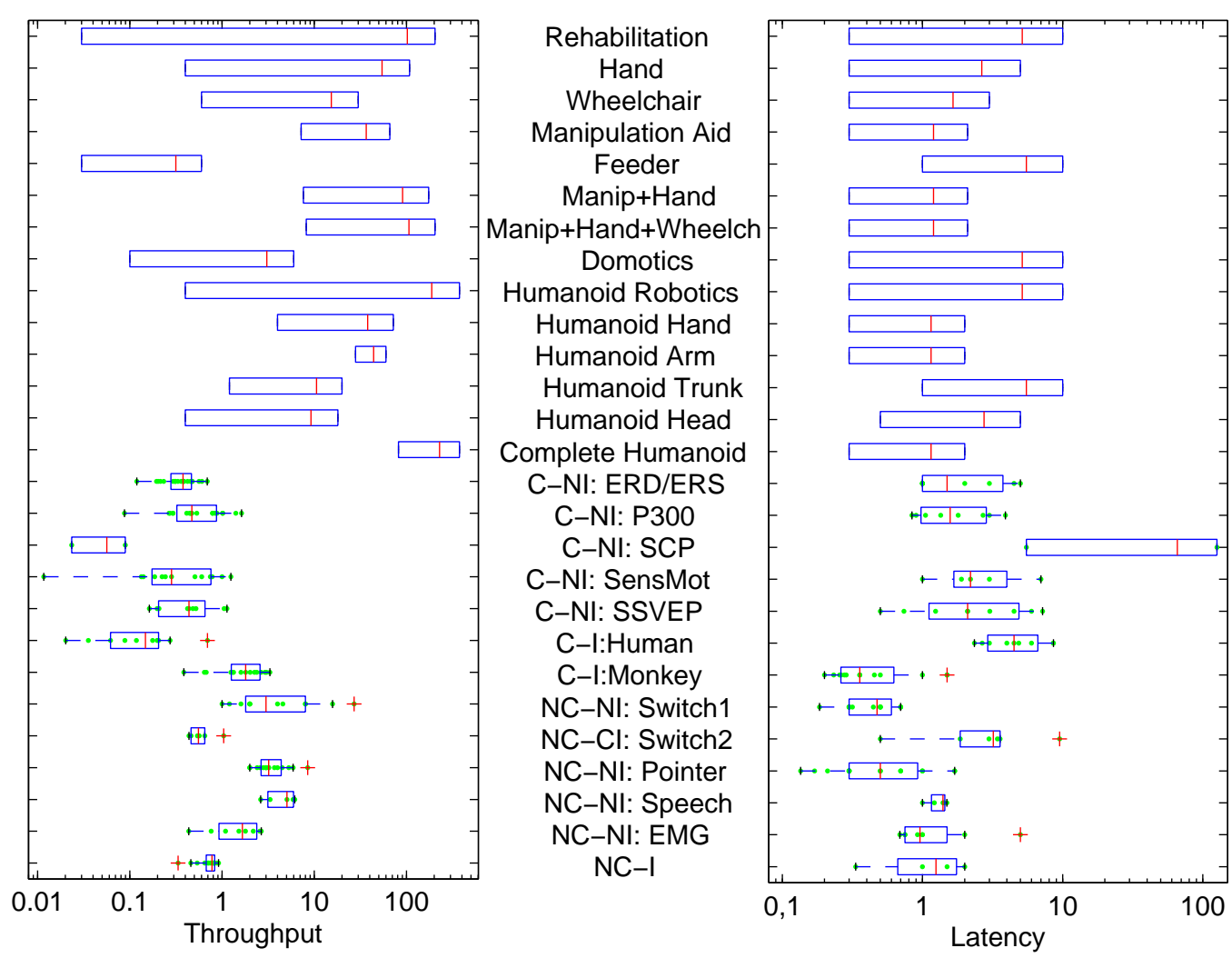

Fig. 7. 


\begin{tabular}{|l|r|r|r|r|r|r|}
\hline \multirow{2}{*}{ Devices } & \multicolumn{3}{|c|}{ Throughput (bit/s) } & \multicolumn{3}{c|}{ Latency (s) } \\
\cline { 2 - 7 } & \multicolumn{1}{|c|}{$\min$} & \multicolumn{1}{|c|}{ median } & \multicolumn{1}{c|}{$\max$} & \multicolumn{1}{c|}{ in } & median & max \\
\hline Rehabilitation & $\mathbf{0 . 0 3}$ & $\mathbf{1 0 2 . 0 2}$ & $\mathbf{2 0 4 . 0 0}$ & $\mathbf{0 . 3 0}$ & $\mathbf{5 . 1 5}$ & $\mathbf{1 0 . 0 0}$ \\
\hline Hand & 0.40 & 54.20 & 108.00 & 0.30 & 2.65 & 5.00 \\
\hline Wheelchair & 0.60 & 15.30 & 30.00 & 0.30 & 1.65 & 3.00 \\
\hline Manipulation aid & 7.20 & 36.60 & 66.00 & 0.30 & 1.20 & 2.10 \\
\hline Feeder & 0.03 & 0.32 & 0.60 & 1.00 & 5.50 & 10.00 \\
\hline Manipulator+Hand & 7.60 & 90.80 & 174.00 & 0.30 & 1.20 & 2.10 \\
\hline Manipulator+Hand+Wheelchair & 8.20 & 106.10 & 204.00 & 0.30 & 1.20 & 2.10 \\
\hline Domotics & $\mathbf{0 . 1 0}$ & $\mathbf{3 . 0 5}$ & $\mathbf{6 . 0 0}$ & $\mathbf{0 . 3 0}$ & $\mathbf{5 . 1 5}$ & $\mathbf{1 0 . 0 0}$ \\
\hline Humanoid robotics & $\mathbf{0 . 4 0}$ & $\mathbf{1 8 7 . 7 0}$ & $\mathbf{3 7 5 . 0 0}$ & $\mathbf{0 . 3 0}$ & $\mathbf{5 . 1 5}$ & $\mathbf{1 0 . 0 0}$ \\
\hline Humanoid Hand & 4.00 & 38.00 & 72.00 & 0.30 & 1.15 & 2.00 \\
\hline Humanoid Arm & 28.00 & 44.00 & 60.00 & 0.30 & 1.15 & 2.00 \\
\hline Humanoid Trunk & 1.20 & 10.60 & 20.00 & 1.00 & 5.50 & 10.00 \\
\hline Humanoid Head & 0.40 & 9.20 & 18.00 & 0.50 & 2.75 & 5.00 \\
\hline Complete Humanoid & 82.00 & 228.50 & 375.00 & 0.30 & 1.15 & 2.00 \\
\hline
\end{tabular}

Table 1 


\begin{tabular}{|l|r|r|r|r|r|r|}
\hline \multirow{2}{*}{ Interfaces } & \multicolumn{3}{|c|}{ Throughput (bit/s) } & \multicolumn{3}{|c|}{ Latency (s) } \\
\cline { 2 - 7 } & \multicolumn{1}{|c|}{ min } & median & max & min & median & max \\
\hline Cortical non-invasive (C-NI) & $\mathbf{0 . 0 1}$ & $\mathbf{0 . 4 2}$ & $\mathbf{1 . 6 3}$ & $\mathbf{0 . 5 0}$ & $\mathbf{2 . 1 0}$ & $\mathbf{1 2 6 . 0 0}$ \\
\hline ERD/ERS & 0.12 & 0.38 & 0.69 & 1.00 & 1.50 & 5.00 \\
\hline P300 & 0.09 & 0.47 & 1.63 & 0.84 & 1.58 & 3.90 \\
\hline SCP & 0.02 & 0.06 & 0.09 & 5.50 & 65.75 & 126.00 \\
\hline Sensorimotor (SensMot) & 0.01 & 0.28 & 1.25 & 1.00 & 2.20 & 7.00 \\
\hline SSVEP & 0.16 & 0.44 & 1.13 & 0.50 & 2.10 & 7.20 \\
\hline Cortical invasive (C-I): Human & $\mathbf{0 . 0 2}$ & $\mathbf{0 . 1 5}$ & $\mathbf{0 . 7 0}$ & $\mathbf{2 . 3 5}$ & $\mathbf{4 . 5 0}$ & $\mathbf{8 . 6 0}$ \\
\hline Cortical invasive (C-I): Monkey & $\mathbf{0 . 3 9}$ & $\mathbf{1 . 8 0}$ & $\mathbf{3 . 3 0}$ & $\mathbf{0 . 2 0}$ & $\mathbf{0 . 3 6}$ & $\mathbf{1 . 5 0}$ \\
\hline Non-cortical non-invasive (NC-NI) & $\mathbf{0 . 4 4}$ & $\mathbf{2 . 6 1}$ & $\mathbf{2 7 . 0 2}$ & $\mathbf{0 . 1 4}$ & $\mathbf{0 . 7 0}$ & $\mathbf{9 . 5 0}$ \\
\hline Switch1 & 1.00 & 3.00 & 27.02 & 0.18 & 0.47 & 0.70 \\
\hline Switch2 & 0.44 & 0.55 & 1.04 & 0.50 & 3.20 & 9.50 \\
\hline Pointer & 2.00 & 3.20 & 8.50 & 0.14 & 0.50 & 1.70 \\
\hline Speech & 2.63 & 5.04 & 6.13 & 1.00 & 1.40 & 1.49 \\
\hline EMG & 0.44 & 1.66 & 2.66 & 0.69 & 0.96 & 5.00 \\
\hline Non-cortical invasive (NC-I) & $\mathbf{0 . 3 3}$ & $\mathbf{0 . 7 8}$ & $\mathbf{0 . 9 2}$ & $\mathbf{0 . 3 3}$ & $\mathbf{1 . 2 5}$ & $\mathbf{2 . 0 0}$ \\
\hline
\end{tabular}

Table 2 